Original Paper http://ajol.info/index.php/ijbcs http://indexmedicus.afro.who.int

\title{
Importance socio-économique de la prune rouge (Haematostaphis barteri Hook F.) au Bénin
}

\author{
Bienvenue Nawan SOUROU ${ }^{1 *}$, Jacob YABI ${ }^{2}$, \\ Christine Ajokè Ifetayo Nougbodé OUINSAVI ${ }^{1}$ et Nestor SOKPON ${ }^{1}$ \\ ${ }^{1}$ Faculty of Agronomy, Laboratory of Forestry Studies and Research, \\ University of Parakou,BP 123 Parakou, Benin. \\ ${ }^{I}$ Faculté d'Agronomie, Laboratoire d'Etudes et de Recherches Forestières (FA/LERF), \\ Université de Parakou, BP : 123 Parakou, Bénin. \\ ${ }^{2}$ Département d'Economie et de Sociologie Rurales, Faculté d'Agronomie, \\ Université de Parakou, BP 123, Parakou, Bénin. \\ *Auteur correspondant ; E-mail : bienvenuesourou@yahoo.fr; BP : 315 Natitingou, Tél : (+229) 97242352.
}

\section{RESUME}

L'importance socio-économique de H. barteri a été étudiée au Nord-Ouest du Bénin sur la base d'enquêtes ethnobotanique et socio-économique administrées respectivement sur 230 et 100 personnes des deux sexes et choisies au hasard dans les trois groupes socioculturels majoritaires du milieu d'étude à savoir les Waaba, les Bètammaribè et les Natimba. Le modèle de COBBDOUGLAS a été utilisé pour évaluer les déterminants du profit net annuel obtenu de la vente des fruits de $H$. barteri. L'étude a montré que les principaux organes de l'espèce à savoir les feuilles, les racines et les écorces sont employés dans le traitement de plusieurs maladies alors que les fruits sont essentiellement utilisés comme aliment. Ces différentes formes d'utilisations de H. barteri varient d'un groupe ethnique à un autre. Le revenu moyen annuel obtenu de la vente des fruits de H. barteri est faible (10 077 FCFA) et est significativement $(\mathrm{P}<0,001)$ influencé par la quantité totale de mains d'œuvre employée et la quantité de capital total utilisée. La conservation in situ des arbres de $H$. barteri et la valorisation de ses fruits à travers leur transformation pourraient contribuer à la préservation future de l'espèce.

(C) 2016 International Formulae Group. All rights reserved.

Mots clés : Bénin, conservation, ethnobotanique, Haematostaphis barteri, rentabilité économique. 


\title{
Socio-economic importance of Blood plum (Haematostaphis barteri Hook F.) in Benin
}

\begin{abstract}
The socioeconomic importance of $H$. barteri has been studied in North West Benin on the basis of ethnobotanical and socioeconomic investigations respectively administered to 230 and 100 people of both sexes and randomly selected from the three majority sociocultural groups of study area namely Waaba, Bètammaribé and Natimba. The COBB-DOUGLAS model has been used to assess the determinants of annual net profit obtained from the sale of $H$. barteri fruits. The study shows that the main organs of the species namely the leaves, roots and barks are used in the treatment of many diseases while fruits are mainly used as food. These different uses of $H$. barteri vary from one ethnic group to another. The average annual income obtained from the sale of $H$. barteri fruits is low (10 077 FCFA) and is significantly $(\mathrm{P}<0.001)$ influenced by the total workforce and the amount of total capital used. In situ conservation of $H$. barteri trees and the valorization of its fruits through their transformation could contribute to the future preservation of the species.
\end{abstract}

(C) 2016 International Formulae Group. All rights reserved.

Keywords: Benin, conservation, ethnobotany, Haematostaphis barteri, economic profitability.

\section{INTRODUCTION}

En Afrique, les ressources génétiques forestières et en particulier les arbres fruitiers jouent un rôle important dans la résolution des problèmes des populations rurales aussi sur les plans agricole, alimentaire, socioéconomique et médicinal. Elle procure à travers la vente des revenus aux ménages et participent de la sécurité alimentaire et à l'amélioration de la santé et de l'état nutritionnel de ces populations grâce à une importante diversité (Bowe et Haq, 2010 ; Dan Guimbo et al., 2012). Ces plantes sont riches en nutriments et possèdent de nombreuses vertus médicinales. La conservation de cette biodiversité forestière permettra de maintenir leur rôle de production, de protection écologique et de satisfaction des besoins de la population (World Agroforestry Centre, 2008). Malgré cette richesse de la biodiversité en Afrique, les populations restent confrontées aux problèmes liés à la sécurité alimentaire. La résolution de ces problèmes passera par l'amélioration des pratiques culturales, l'agriculture favorisant les ressources naturelles et le développement de nouveaux systèmes de production qui permettront d'augmenter de façon durable la productivité de ces ressources naturelles tout en sauvegardant le capital environnemental pour les générations futures (Assogbadjo, 2006). Ceci nécessite une meilleure connaissance du potentiel des ressources naturelles disponibles et déjà intégrées dans la culture des populations rurales.

Haematostaphis barteri est l'une de ces ressources génétiques forestières qui appartient à la grande famille des anacardiaceae. Elle n'existe qu'en Afrique tropicale (Côte d'Ivoire, Ghana, Togo, Bénin, Cameroun et Tchad) et se rencontre dans les savanes boisées, surtout sur les collines rocheuses et éboulées en zone soudanienne et guinéenne (Arbonnier, 2002). Au Bénin, les 
peuplements de $H$. barteri sont présents sur la chaîne de l'Atacora, au Nord-Ouest du département de l'Atacora (Akoègninou et al., 2006 ; Sourou et al., 2016).

Plusieurs études ont été menées au Bénin et ailleurs sur "les valeurs d'usage" de différentes espèces : Assogbadjo et al. (2010) sur Caesalpinia bonduc ; Gouwakinnou et al., (2011) sur Sclerocarya birrea; Makalao et al. (2015) sur Parkia biglobosa, Hyphaene thebaïca, Balanites aegyptiaca, Tamarindus indica. Parmi les différents travaux scientifiques réalisés au Bénin et dans la sousrégion, ceux sur $H$. barteri sont rares. Cependant, quelques travaux ont été effectués sur $H$. barteri notamment sur la composition chimique de ses principaux organes et sur la valeur nutritive de ces fruits au Nigéria et au Ghana (Kubmarawa et al., 2000 ; Eromosele et Eromosele, 2002; Asase et al., 2005; Umaru et al., 2007 ; Tadzabia et al., 2013) et sur son écologie au Bénin et au Togo (Agbogan et al., 2012 ; Agbogan et al., 2015 ; Sourou et al., 2016). Il importe ainsi de mener des recherches scientifiques sur l'importance socio-économique de $H$. barteri afin d'établir des stratégies de conservation pour une utilisation durable de cette ressource naturelle indigène.

Compte tenu de l'importance de l'espèce pour les communautés rurales (Eyana et al., 2007) et les insuffisances signalées à sa valorisation et à sa domestication surtout à cause d'un manque de connaissances ethnobotaniques (Eyog Matig et al., 2002), il importe d'élargir et d'approfondir les connaissances pour sa valorisation économique, son utilisation et sa gestion ultérieure plus durable in situ et ex situ dans les systèmes agroforestiers traditionnels. En conséquence, la présente étude s'accorde avec la préoccupation du gouvernement béninois en matière de prise en compte des savoirs locaux dans la gestion des ressources naturelles (MEHU, 2002).
L'étude a donc pour objectif principal, d'évaluer l'importance socio-économique de $H$. barteri en vue de son intégration future dans une économie formelle au Bénin. De façon spécifique, l'étude vise à: (i) inventorier les différentes connaissances et utilisations de $H$. barteri par les populations locales, (ii) évaluer la rentabilité économique de la vente des fruits de $H$. barteri.

\section{MATERIEL ET METHODES \\ Présentation du cadre d'étude}

La présente étude est réalisée dans trois communes (Natitingou, Boukoumbé et Toucountouna) situées au Nord-Ouest du Bénin entre d'une part les parallèles $10^{\circ}$ et $10^{\circ} 35^{\prime}$ latitude Nord et, d'autre part entre les méridiens $1^{\circ} 06^{\prime}$ et $1^{\circ} 30^{\prime}$ longitude Est (Figure 1). Le milieu d'étude est sous l'influence du climat soudanien avec deux saisons bien tranchées: une saison pluvieuse de mi-mai à mi-octobre et une saison sèche de mi-octobre à mi-mai (Tchabi et Adechi, 2014). Les précipitations annuelles varient entre 1000 et $1200 \mathrm{~mm}$. Deux types de sol caractérisent le milieu d'étude: Les sols ferrugineux tropicaux lessivés concrétionnés, à texture limono-sableuse, très poreux et les sols ferrugineux tropicaux lessivés hydromorphes en profondeur. La zone d'étude est caractérisée par une mosaïque de végétations constituées de forêts, d'ilots de forêts galerie et de savanes. Trois principaux groupes socio-linguistiques coexistent dans le milieu d'étude. Il s'agit des Waaba qui représentent $11,2 \%$ de la population du Nord Bénin, des Bètammaribè $4,9 \%$ de la population du nord Bénin et des Natimba 9,9\% de la population du Nord Bénin (INSAE, 2002). A ces principaux groupes s'ajoutent les Dendi, les Bariba et les peulhs. Les populations de ces trois communes sont essentiellement rurales (67\%), et seulement $15 \%$ sont alphabétisées (INSAE, 2002). L'agriculture et l'élevage sont les principales activités des populations actives. 
Méthode d'échantillonnage et collecte des données Aspects ethnobotaniques

L'étude ethnobotanique s'est déroulée dans différentes localités (villages) situées dans trois communes. Dans chacune de ces communes, le choix des localités d'étude (villages) a été basé sur deux critères. Le premier critère est la présence et l'abondance relative des individus de l'espèce $H$. barteri dans les formations végétales de la localité. Le deuxième critère de choix des localités d'étude était relatif à l'existence dans ces dernières, de marchés où les divers organes et particulièrement les fruits de $H$. barteri sont commercialisés, ceci pour s'assurer de l'importance économique de l'arbre pour les populations locales. En tenant compte des critères ci-dessus énumérés, 12 villages répartis dans l'ensemble des trois communes ont été considérés sur environ 118 villages au total (Tableau 1). La taille de l'échantillon pour l'étude a été obtenue en utilisant l'approximation normale de la distribution binomiale proposée par Dagnelie (1998) :

$$
\mathrm{N}=\boldsymbol{\mu}_{1-\alpha / 2}^{2} \frac{P i(1-P i)}{\delta^{2}}
$$

$P i(82 \%)$ a été déterminé à partir de l'enquête exploratoire réalisée dans une localité d'étude par commune et représente la proportion des personnes qui ont utilisé une fois les organes du $H$. barteri. $\boldsymbol{\mu}_{\mathbf{1 - \alpha} / \mathbf{2}}=1,96$, représente la valeur de la variable aléatoire normale pour un risque $\alpha$ égal à 0,05 . La marge d'erreur $\delta$ prévue pour tout paramètre à estimer à partir de l'enquête est de $5 \%$. La taille $\mathrm{N}$ de l'échantillon est ainsi sensiblement égale à environ 230 individus. Le nombre de personnes interrogées par localité a été déterminé par proportionnalité en considérant la taille de la population de chaque localité.

L'enquête a été réalisée entre septembre à octobre 2013 et juillet 2015. Dans chaque commune, un focus group de 15 à 25 personnes des deux sexes a d'abord été réalisé pour recenser les réponses collectives sur les différentes informations recherchées. A l'issue $\mathrm{du}$ focus group, la liste des différentes questions a été établie pour l'enquête individuelle. Les enquêtés ont été choisis au hasard dans chaque localité (Tableau 1). Les interviews ont été conduites en présence d'un traducteur en cas de besoin. Les données collectées auprès de chaque enquêté aussi bien quantitatives que qualitatives sont relatives aux caractéristiques sociodémographiques de l'enquêté, les différentes utilisations de $H$. barteri, les organes utilisés, les fréquences d'utilisation, les lieux d'approvisionnement, les fréquences d'approvisionnement et le mode d'acquisition (achat ou cueillette, etc.). Les données relatives aux connaissances sur l'habitat et le mode de gestion de l'espèce ont été également collectées.

\section{Aspects économiques}

L'enquête économique a été réalisée sur les marchés de Natitingou, de Boukoumbé et de Wansokou (dans la commune de Toucountouna) qui constituent les seuls marchés du milieu d'étude où la vente des fruits de $H$. barteri est observée chaque année de juin à août. La taille de l'échantillon a été obtenue en utilisant également l'approximation normale de la distribution binomiale proposée par Dagnelie (1998) et indiquée plus haut. A partir des valeurs de $\mathrm{p}$ issues des résultats de la phase exploratoire de l'étude, au total 100 vendeurs des fruits de $H$. barteri ont été sélectionnés dans le milieu d'étude. Ils ont été répartis dans les trois marchés presque équitablement. Ainsi, 35 vendeurs sur chacun des marchés de Natitingou et de Boukoumbé et 30 vendeurs dans celui de Wansokou ont été sélectionnés au hasard.

Les données de cet aspect économique de étude ont été collectées de juin à août 2014 à l'aide d'un questionnaire et a permis de recueillir des informations quantitatives et qualitatives relatives aux caractéristiques socio-économiques des vendeurs de fruits de $H$. barteri, l'identification des acteurs 
impliqués dans la commercialisation, l'identification des circuits de commercialisation, la quantité de fruits collectés, le prix de vente des fruits et le coût des différentes charges liées à cette activité. Les fruits de $H$. barteri étant vendus par petits tas à 25 FCFA, 150 tas de fruits ont été pesés durant toute la saison de vente (soit 50 tas en début de saison, 50 tas en période d'abondance et 50 tas vers la fin de la saison) auprès de 10 vendeurs choisis au hasard dans chacun des trois marchés. Ces mesures ont permis d'estimer la masse moyenne en kilogramme d'un tas de 25 FCFA dans chacun des marchés.

\section{Analyses statistiques}

\section{Aspect ethnobotanique}

Pour le traitement des données, les informations collectées ont été compilées par groupe sociolinguistique, par village et par sexe grâce au tableur Microsoft Excel. Le taux de réponse par type d'utilisation a été exprimé par la formule utilisée par Gouwakinnou (2011), et qui se présente comme suit: $\mathbf{F}=\mathbf{S} / \mathbf{N} \times 100$ avec,

S: nombre de personnes ayant fourni une réponse par rapport à une utilisation donnée; et

$\mathrm{N}$ : nombre total de personnes interviewées.

Le $\mathrm{khi}^{2}\left(\mathrm{X}^{2}\right)$ a été déterminé afin de vérifier s'il y a un lien ou non entre les groupes sociolinguistiques et les différents usages faits des différents organes de $H$. barteri grâce au logiciel SPSS 16.0. Par ailleurs, une Analyse Factorielle des Correspondances (AFC) a été réalisée sur la matrices de données correspondant aux fréquences absolues des réponses des interviewés sur les différentes organes de l'espèce utilisés afin de mieux décrire les relations existantes entre les organes de $H$. barteri les plus utilisés et les groupes sociolinguistiques les plus concernés grâce au logiciel Minitab 14.

\section{Aspects économiques}

Après codification dans le tableur Microsoft Excel, les données d'enquête ont été analysées à l'aide du logiciel SPSS version 16.0 pour la détermination des statistiques descriptives en termes de pourcentage et de moyenne. Ces données quantitatives ont été ensuite soumises à une analyse de variance (ANOVA) en utilisant la procédure PROC GLM du logiciel SPSS version 16.0 et les comparaisons de moyennes multiples ont été réalisées avec le test de Student NewmanKeuls.

En se basant sur les considérations théoriques et en s'appuyant sur les travaux de Gnanglè et al. (2012) et de Balogoun et al. (2014), il est possible d'écrire le modèle de la rentabilité de la vente des fruits de $H$. barteri sous la forme de l'équation de COBBDOUGLAS défini comme suit :

$$
R N_{i}=e^{\alpha_{0}} \quad Q M O_{i}^{k 1} C A P I_{i}^{k 2}\left(\sum_{p=1}^{n} Z_{i}^{\beta}\right)
$$

En appliquant la fonction logarithme népérien à chaque membre de l'équation (1), on obtient :

$$
\ln \left(R N_{i}\right)=\alpha_{0}+k_{1} \ln \left(Q M 0_{i}\right)+k_{2} \ln \left(C A P I_{i}\right)+\sum_{p=1}^{7} \beta Z_{i}+\varepsilon_{i}
$$

$\mathrm{RN}_{\mathrm{i}}$ est le revenu net ou marge nette du vendeur i en FCFA. Il est calculé par la formule mathématique suivante: $\mathbf{R N}_{\mathrm{i}}=\mathbf{P B V}_{\mathrm{i}}-\mathrm{CT}_{\mathrm{i}}=\mathrm{PBV}_{\mathrm{i}}-\mathrm{CV}_{\mathrm{i}}-\mathrm{CF}_{\mathrm{i}}$ (3)

Ici, PBVi est le produit brut en valeur, donné par la quantité total de fruits (en $\mathrm{Kg}$ ) collectés par le vendeur i pendant toute la période de collecte multiplié par le prix unitaire d'un kilogramme de fruits de $H$. barteri, CVi est l'ensemble des charges variables du vendeur i constituées ici essentiellement par les coûts de la main d'œuvre de collecte de fruits, CFi est l'ensemble des charges fixes du vendeur $\mathrm{i}$ formées ici principalement du coût de droit de place au marché et CTi (coût total) correspondant à la somme des coûts variables 
et des coûts fixes. Les variables QMOi et CAPIi représentent respectivement pour le vendeur, la quantité totale de main d'œuvre utilisée en homme-jour et la quantité totale de capital utilisé en FCFA (main d'œuvre rémunérée, frais de transport vers le marché, droit de place sur le marché, etc.). Les Zi sont les variables relatives aux caractéristiques socioéconomiques du vendeur $\mathrm{i}$ et des marchés. Les coefficients $\alpha$, $\mathrm{k}$ et $\beta$ sont les paramètres à estimer et $\varepsilon_{\mathrm{i}}$ sont les termes d'erreur.

Le modèle a été estimé par la méthode des Moindres Carrés Ordinaires (MCO). De plus, des tests de Durbin-Waston, de Goldfeld et Quandt et de Farrar-Glauber ont été réalisés afin de vérifier respectivement si des erreurs d'autocorrélation, d'hétérocédasticité et de multicolinéarité existent et sont significatives. En particulier, les erreurs de multicolinéarité ont été corrigées par la méthode d'estimation par étapes qui élimine au fur et à mesure les variables fortement corrélées à d'autres dans le modèle jusqu'à l'obtention d'une estimation sans erreur de multicolinarité.

\section{RESULTATS}

\section{Connaissances ethnoécologiques de $\boldsymbol{H}$.} barteri

Appellation locale de $\mathrm{H}$. barteri et fondement

Haematostaphis barteri couramment appellé prune rouge en français et blood plum en anglais, porte différents noms locaux suivant les groupes socio-lingustiques. Le nom local de «Sanmoru» chez les Dendi et «éyégninrè » chez les bètammaribè signifie acide, qui correspond à la description du goût des fruits tel que perçu par les populations locales. Le nom « tangninringnirimbou » chez les Waaba se rapporte à l'habitat naturel de l'espèce identifié par ces populations comme étant les collines rocheuses. Chez les Natimba, le nom local «Tangnayibou» combine à la fois le goût acidulé des fruits et l'habitat naturel de l'espèce (collines rocheuses).

\section{Connaissances ethnobotaniques utilisations de $\boldsymbol{H}$. barteri au Bénin}

et

Dans la zone d'étude, différentes formes d'utilisation sont faites de H. barteri à savoir : usage alimentaire, usage médicinal et économique (Tableau 2). La plupart des formes d'utilisation de $H$. barteri varient significativement en fonction des groupes socio-linguistiques (Tableau 2). Les principaux organes de l'espèce sont utilisés mais à des degrés divers : $31 \%$ des enquêtés utilisent les fruits, $28 \%$ utilisent les feuilles, $19 \%$ utilisent les racines et les écorces et 3\% font usage des tiges (Figure 2). De même, les organes de l'espèce utilisés par les populations locales varient en fonction des ethnies $\left(\mathrm{X}^{2}=32,217 \mathrm{ddl}=2 ; \mathrm{p}=0,000\right.$ pour les feuilles, $\mathrm{X}^{2}=178,618 ; \mathrm{ddl}=2 ; \mathrm{p}=0,000$ pour les racines et $X^{2}=205,564 \mathrm{ddl}=2$; $\mathrm{p}=0,000$ pour les écorces).

L'Analyse Factorielle des Correspondances (AFC) effectuée montre qu'avec les deux premiers axes, on explique $100 \%$ de l'information sur les différents organes utilisés par les groupes sociolinguistiques. La Figure 3 donne une projection des différents organes utilisés et les groupes socio-linguistiques sur les deux premiers axes factoriels. L'analyse de cette figure révèle que les Waaba et les Natimba utilisent beaucoup plus les racines et l'écorce alors que les Bètammaribè utilisent plus les feuilles et les fruits.

Les Waaba utilisent beaucoup plus les écorces et les racines de $H$. barteri pour traiter les morsures de serpents et d'araignées, la varicelle, les douleurs des côtes et de la hanche, les entorses et pour lutter contre l'asthénie. Les Natimba, quant à eux, utilisent les mêmes organes pour traiter les hépatites, les hémorroïdes, les maux de ventre, l'anémie, la fièvre et les convulsions. Par contre, les Bètammaribè utilisent beaucoup plus les feuilles pour traiter le paludisme. Les fruits sont utilisés de façon homogène (100\%) par les trois groupes socio-culturels dans 
l'alimentation. La pulpe des fruits mûrs est directement consommée comme aliment (Tableau 2). La majorité des populations (98\%) affirment que les organes de $H$. barteri ne font l'objet d'aucune transformation. Néanmoins, chez les Natimba $(29,1 \%)$ et les Bètammaribè (19,7\%), les enfants fabriquent la boisson $d$ 'H. barteri par simple extraction du jus de la pulpe des fruits auquel ils ajoutent du sucre ou de la cendre. Ladite boisson est destinée à la consommation familiale et non à la commercialisation.

Importance économique de $\boldsymbol{H}$. barteri Caractéristiques socio-économique des vendeurs et quelques éléments de leurs comptes d'exploitation

Les fruits de $H$. barteri font l'objet de commercialisation au niveau local. Les principaux acteurs de cette commercialisation sont des femmes (97\%) des villages, qui collectent directement les fruits de $H$. barteri dans les formations végétales et les vendent dans les marchés des chefs lieux de communes sauf à Toucountouna où ils ne sont vendus qu'au marché de Wansokou. Ces vendeurs des fruits de $H$. barteri appartiennent essentiellement au groupe socio-linguistique des Bètammaribè (73\%) et les $27 \%$ restant appartiennent aux groupes socio-lingustiques des Waaba et des Natimba. Il n'existe aucun instrument local de mesure pour cette commercialisation. Les fruits récoltés avec leurs pédoncules sont vendus en de petits tas à 25 FCFA. Les différentes pesées effectuées ont permis d'estimer la masse moyenne d'un tas de fruits de $H$. barteri à 216,903 g sur le marché de Natitingou; 343,285 g sur le marché wansokou et 507,428 g sur le marché de Boukoumbé.

Le Tableau 3 présente des éléments du compte des enquêtés. L'analyse de ce tableau révèle que la quantité moyenne de fruits collectés, le prix de vente moyen d'un kilogramme de fruits, les charges totales moyennes, la marge nette moyenne sur un kilogramme de fruits vendus et le profit annuel moyen des enquêtés du marché de Natitingou sont nettement plus élevés que ceux des vendeurs des marchés de Boukoumbé et de Wansokou (Toucountouna) dont les comptes de vente sont presque similaires. Cependant, la quantité moyenne de fruits collectés par les vendeurs du marché de Wansokou est plus faible avec une marge nette moyenne plus élevée que ceux des vendeurs du marché de Boukoumbé. Le profit moyen annuel issu de la vente des fruits de $H$. barteri est de 10077 FCFA dans la zone d'étude.

Déterminants du profit net généré par la vente des fruits de $\mathrm{H}$. barteri

Les résultats du modèle de régression estimé pour identifier les déterminants du profit net du vendeur des fruits de $H$. barteri sont présentés dans le Tableau 4. L'analyse de ce tableau montre que le modèle de régression est hautement significatif ( $\mathrm{p}<0,001)$. Ainsi, $88 \%$ des variations du profit net annuel des vendeurs enquêtés sont expliqués par les variations des variables introduites dans le modèle. Les $12 \%$ de variations du profit net annuel non expliquées par les variations des variables introduites dans le modèle seraient attribuables aux facteurs difficilement mesurables tels que le niveau de fertilité des sols, les conditions climatiques et les divers changements qu'on peut enregistrer d'une saison à l'autre.

Les résultats obtenus indiquent que la quantité de main d'œuvre a des effets positifs et hautement significatifs ( $\mathrm{p}<0,001$ ) sur le profit net du vendeur. Le coût total des charges a par contre un effet négatif hautement significatif $(\mathrm{p}<0,001)$. Pour les variables socio-économiques mesurées, seuls le marché de Natitingou et l'appartenance du vendeur au groupe socio-linguistique des Waaba ont un effet positif et hautement significatif $(\mathrm{p}<0,001)$ sur le profit annuel du vendeur. 


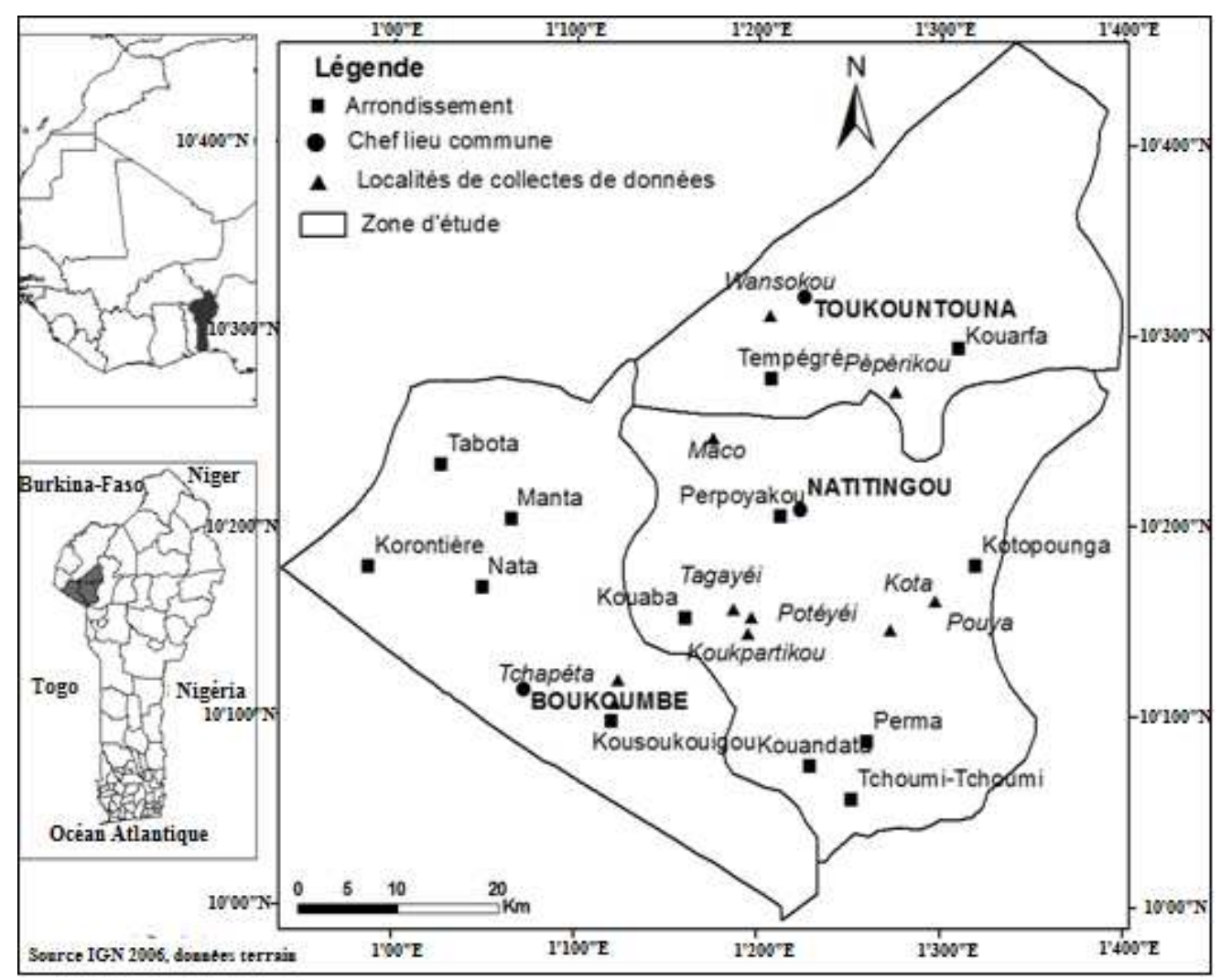

Figure 1 : Carte de localisation de la zone d'étude.

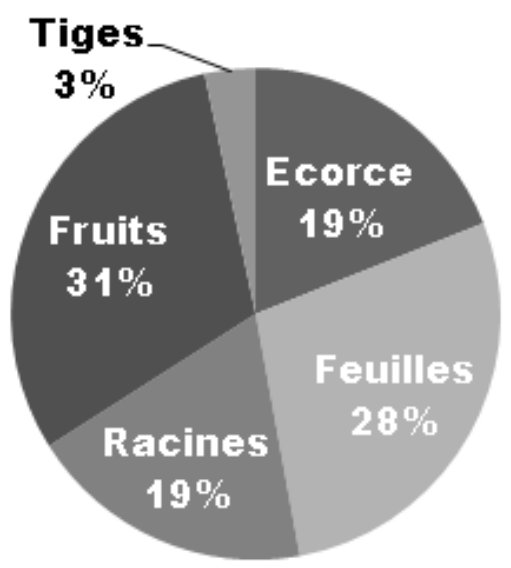

Figure 2: Répartition des différents organes de $H$. barteri en fonction de leurs fréquences d'utilisation par les populations locales. 


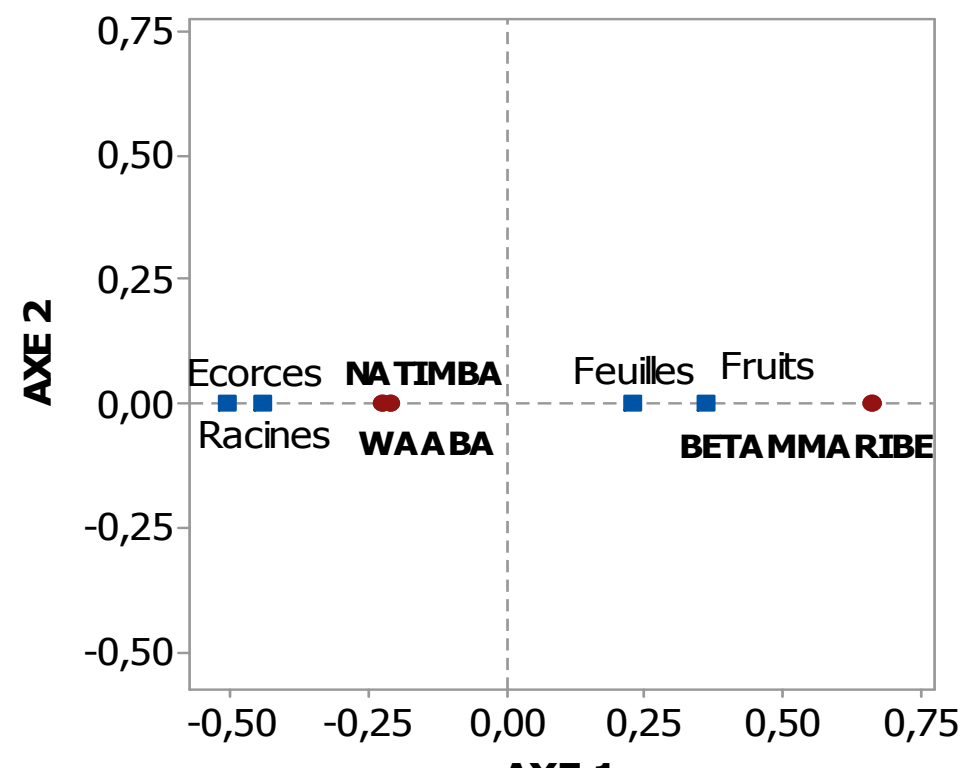

AXE 1

Figure 3: Représentation graphique des différents organes de H. barteri utilisés par les ethnies sur les axes factoriels 1 et 2 .

Tableau 1 : Taille de l'échantillon.

\begin{tabular}{|c|c|c|c|c|c|}
\hline \multirow{2}{*}{ Communes } & \multirow{2}{*}{ Villages } & \multirow{2}{*}{$\begin{array}{c}\text { Groupes socio- } \\
\text { linguistiques }\end{array}$} & \multicolumn{2}{|c|}{ Echantillon } & \multirow{2}{*}{ Total } \\
\hline & & & Hommes & Femmes & \\
\hline \multirow{7}{*}{ Natitingou } & Kota & \multirow{2}{*}{ Waaba } & 8 & 7 & 15 \\
\hline & Pouya & & 10 & 5 & 15 \\
\hline & Koussantikou & \multirow{5}{*}{ Bètammaribè } & 5 & 5 & 10 \\
\hline & Koukpartikou & & 6 & 4 & 10 \\
\hline & Potéyéi & & 4 & 6 & 10 \\
\hline & Tagayéi & & 6 & 4 & 10 \\
\hline & Kouaba & & 4 & 6 & 10 \\
\hline \multirow{2}{*}{ Boukoumbé } & Tchapéta & \multirow{2}{*}{ Bètammaribè } & 5 & 5 & 10 \\
\hline & Koussoukouingou & & 16 & 14 & 30 \\
\hline \multirow{5}{*}{ Toucountouna } & Pèpèrikou & \multirow{4}{*}{ Waaba } & 6 & 4 & 10 \\
\hline & Tampégré & & 4 & 6 & 10 \\
\hline & Maco & & 5 & 5 & 10 \\
\hline & \multirow{2}{*}{ Wansokou } & & 11 & 9 & 20 \\
\hline & & Natimba & 28 & 32 & 60 \\
\hline Total & & & 118 & 112 & 230 \\
\hline
\end{tabular}


Tableau 2 : Les différentes utilisations de $H$. barteri.

\begin{tabular}{|c|c|c|c|c|c|c|}
\hline \multirow{2}{*}{ Organes } & \multirow{2}{*}{ Domaine d'utilisation } & \multirow{2}{*}{ Forme d'utilisation } & \multirow{2}{*}{$\begin{array}{l}\text { But de } \\
\text { l'utilisation }\end{array}$} & \multicolumn{3}{|c|}{ Taux de réponse par ethnie } \\
\hline & & & & Waaba $(n=80)$ & Bètammaribè (n = 90) & Natimba $(n=60)$ \\
\hline \multirow{7}{*}{ Feuilles } & \multirow{7}{*}{ Médicinale } & \multirow{7}{*}{ Décoction } & Maux de ventre & - & 11,1 & 86,7 \\
\hline & & & Paludisme & $95^{*}$ & 74,5 & $90 *$ \\
\hline & & & $\begin{array}{l}\text { Morsure de } \\
\text { serpent et } \\
\text { d'araignée }\end{array}$ & 56,3 & 16,7 & - \\
\hline & & & Fièvre & - & - & 83,3 \\
\hline & & & Convulsions & 2,5 & - & 66,7 \\
\hline & & & Varisèle & 47,5 & - & - \\
\hline & & & Hémoroïde & - & - & 56,7 \\
\hline \multirow{8}{*}{ Ecorces } & \multirow{8}{*}{ Médicinale } & \multirow{8}{*}{ Décoction - Poudre } & Malaria & $77,5^{*}$ & 4,4 & $90^{*}$ \\
\hline & & & Anti-vénimeux & 57,5 & 11,1 & - \\
\hline & & & Maux de ventre & - & 13,33 & 86,7 \\
\hline & & & Fièvre & - & - & 83,3 \\
\hline & & & Convulsions & 2,5 & - & 66,7 \\
\hline & & & Hémoroïde & 7,5 & - & 60 \\
\hline & & & Varisèle & 47,5 & - & - \\
\hline & & & Carie dentaitre & $2,5^{*}$ & - & $3,3 *$ \\
\hline Racines & Médicinale & Décoction - Poudre & Malaria & $7,5^{*}$ & $4,5^{*}$ & 26,7 \\
\hline
\end{tabular}


B. N. SOUROU et al. / Int. J. Biol. Chem. Sci. 10(1): 326-343, 2016

\begin{tabular}{|c|c|c|c|c|c|c|}
\hline & & & Anti-vénimeux & 57,5 & 2,2 & - \\
\hline & & & Hémoroïde & 5 & - & 56,7 \\
\hline & & & Asthénie & 47,5 & 4,4 & - \\
\hline & & & Douleur & 57,5 & - & 6,7 \\
\hline & & & Entorses & 47,5 & - & 6,7 \\
\hline & & & Anémie & - & _ & 70 \\
\hline & & & Hépatites & - & - & 46,7 \\
\hline \multirow{2}{*}{ Fruits } & Médicinale & Jus de fruit & Anémie & - & - & 80 \\
\hline & Alimentaire & pulpe & Aliment & $100 *$ & $100 *$ & $100 *$ \\
\hline Tiges & Energétique & _ & Source d'énergie & - & 12 & - \\
\hline
\end{tabular}

Les taux de réponse par ethnie d'une même ligne suivis d'un (*) ne sont pas significativement différents au seuil de 5\% suivant le test khi ${ }^{2}$ de Pearson.

Tableau 3: Compte d'exploitation du vendeur suivant le marché.

\begin{tabular}{|c|c|c|c|c|c|}
\hline Marchés & $\begin{array}{c}\text { Quantité de fruits collectés (kg) sur } 2 \\
\text { mois (juin-juillet 2014) }\end{array}$ & $\begin{array}{l}\text { prix de vente } \\
\quad(\mathrm{fcfa} / \mathrm{kg})\end{array}$ & $\begin{array}{l}\text { Charges totales } \\
\quad(\mathrm{fcfa} / \mathrm{kg})\end{array}$ & $\begin{array}{c}\text { Marge nette (fcfa/kg) sur } 2 \\
\text { mois (juin-juillet 2014) }\end{array}$ & $\begin{array}{l}\text { profit moyen } \\
\text { annuel (fcfa) }\end{array}$ \\
\hline Natitingou & $220,32^{\mathrm{a}}( \pm 13,5)$ & $115,26^{\mathrm{a}}( \pm 0,001)$ & $25,67^{\mathrm{a}}( \pm 3,2)$ & $89,59^{\mathrm{a}}( \pm 3,2)$ & $19154^{\mathrm{a}}( \pm 1204)$ \\
\hline Boukoumbé $\quad(\mathrm{n}=35)$ & $111,58^{\mathrm{b}}( \pm 7,04)$ & $49,27^{\mathrm{b}}( \pm 0,001)$ & $2,94^{\mathrm{b}}( \pm 0,064)$ & $46,33^{\mathrm{c}}( \pm 0,064)$ & $5180^{\mathrm{b}}( \pm 332)$ \\
\hline Toucountouna $(\mathrm{n}=30)$ & $74,15^{\mathrm{c}}( \pm 2,42)$ & $72,43^{\mathrm{b}}( \pm 0,001)$ & $2,79^{\mathrm{b}}( \pm 0,001)$ & $70,03^{\mathrm{b}}( \pm 0,001)$ & $5200^{\mathrm{b}}( \pm 177)$ \\
\hline Total & $138( \pm 8,20)$ & $79,23( \pm 2,81)$ & $10,85( \pm 1,61)$ & $68,58( \pm 2,14)$ & $10077( \pm 795)$ \\
\hline
\end{tabular}


B. N. SOUROU et al. / Int. J. Biol. Chem. Sci. 10(1): 326-343, 2016

Tableau 4: Déterminants du profit net de la vente des fruits de $H$. barteri.

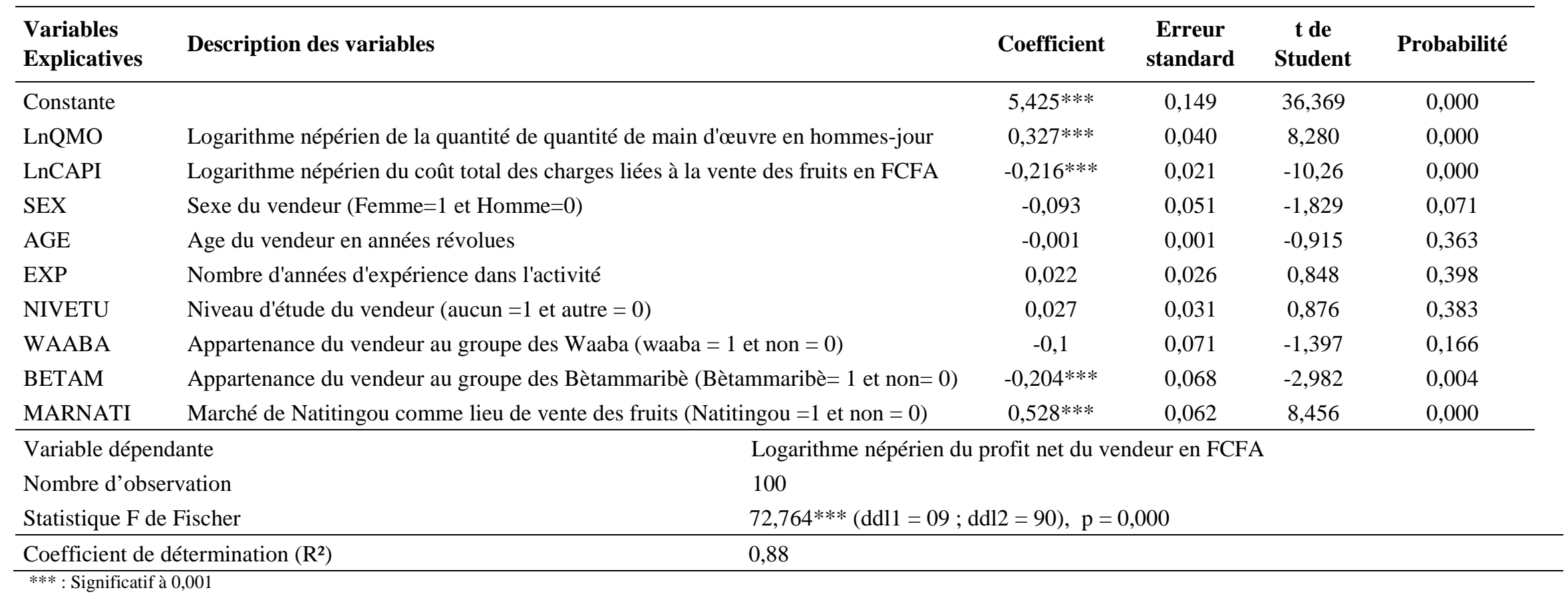




\section{DISCUSSION}

Savoirs ethnoécologiques de $\boldsymbol{H}$. barteri

La majorité des populations locales a indiqué que $H$. barteri semble être bien adapté aux sols gravillonnaires et aux collines rocheuses des savanes. Certains noms vernaculaires de l'espèce révèlent bien cette perception des populations. Ce savoir local concorde bien avec non seulement nos propres observations faites sur le terrain mais aussi avec celles de Arbonnier 2002) dans les zones sèches de l'Afrique de l'Ouest ; Akoègninou et al. (2006) au Bénin; Eyana (2007) et Agbogan et al. (2012) au Togo sur l'habitat de la même espèce.

\section{Importance ethnobotanique de l'espèce}

$H$. barteri est une espèce végétale sauvage dont les principaux organes (racines, écorces, feuilles et fruits) sont employés dans divers usages au Nord-Ouest Bénin (Tableau 2). Les connaissances ethnobotaniques des populations du Nord-Ouest du Bénin sur $H$. barteri sont bien comparables à celles notées par Eyana (2007) au Nord Togo et Asase et al. (2005) au Ghana sur la même espèce. Les propriétés médicinales de $H$. barteri ont été d'ailleurs confirmées grâce aux travaux de Tadzabia et al. (2013) et Boampong et al. (2015) qui ont révélé la présence de certaines substances chimiques (flavonoïdes, saponines et alcaloïdes) dans les racines, les écorces et les feuilles de l'espèce ayant des actions antifongique et antibactérien avérées. Les principes actifs contenus dans ces organes de la plante sont donc consommés par les populations. Cependant, certains rapports ont révélé la présence des composées toxiques dans la plante et dans les graines tels que le tannin $(4,92 \pm 0,34 \mathrm{mg} / 100 \mathrm{~g}$ de feuilles $)$, l'acide phytique $(17,80 \pm 1,80 \mathrm{mg} / 100 \mathrm{~g}$ de feuilles) (Umaru et al., 2007 ; Kubmarawa et al., 2009). A fortes doses, le tannin et l'acide phytique diminuent respectivement la qualité des protéines en réduisant leur digestibilité et l'absorption des minéraux par les animaux y compris l'homme (Kubmarawa et al., 2009). Bien que $H$. barteri contribue à l'amélioration de la santé des populations, il se pose des questions de doses admissibles pour la préserver. Des études de toxicité et pharmacologique plus approfondies doivent donc être entreprises pour contribuer à une meilleure valorisation des connaissances ethnobotaniques sur l'espèce.

Les fruits de $H$. barteri sont exclusivement consommés par les populations locales et donc constituent une source excellente d'éléments nutritifs vitaux. En témoignent les résultats obtenus par Amoo et al. (1999) et Amouzou et al. (2006) sur la composition en éléments nutritifs des fruits de $H$. barteri en général. Ces résultats révèlent que les fruits de $H$. barteri renferment l'azote $(0,23 \%)$, des protéines brutes $(1,5 \%)$; les sucres réducteurs notamment le glucose $(69,5$ $\mathrm{mg} / \mathrm{g})$; le fructose $(71,8 \mathrm{mg} / \mathrm{g})$; le mannose $(76,6 \mathrm{mg} / \mathrm{g})$ et le galactose $(66,9 \mathrm{mg} / \mathrm{g})$, la vitamine $\mathrm{A},(22,401 \mathrm{USP} / 2 \mathrm{~g}$, la vitamine $\mathrm{C}$, (46 $\mathrm{ml} \mathrm{du} \mathrm{mg} / 100)$ et des éléments minéraux $(2,3 \%)$. En effet, la grande période de fructification de cette espèce coïncide avec le début des activités champêtres de la saison pluvieuse (courant mai-juin). Cette période est considérée comme période de soudure dans le département de l'Atacora, marquée par une faible disponibilité alimentaire. La maturation des fruits de $H$. barteri à cette période contribue donc efficacement à l'alimentation des populations.

\section{Importance économique de $\boldsymbol{H}$. barteri}

La vente des fruits de $H$. barteri est menée essentiellement par des femmes des milieux ruraux qui collectent les fruits dans les différentes formations végétales et les vendent le plus souvent les jours de marché aux populations des villes et des grandes agglomérations pour la consommation directe. La vente des fruits de $H$. barteri apparaît économiquement plus rentable pour les vendeurs du marché de Natitingou car la 
marge nette moyenne de ces derniers est significativement plus élevée que celle des vendeurs des deux autres marchés où les fruits sont vendus. Ceci peut s'expliquer par le fait que Natitingou est la plus grande ville du département de l'Atacora où les activités économiques sont développées : notamment, les fruits de $H$. barteri sont utilisés par certaines femmes pour la fabrication d'une boisson naturellement colorée en rouge au vin et vendue frais aux populations.

La marge nette moyenne sur un kilogramme de fruits vendus et le profit annuel moyen du vendeur (Tableau 3) pour une activité couvrant environ deux mois dans l'année ne sont pas négligeables eu égard aux statistiques du service de l'analyse de la sécurité alimentaire du Programme Alimentaire Mondial qui indiquent que la médiane des dépenses du ménage par personne par mois est de moins de 10000 CFA dans l'Atacora en 2013 (Programme Alimentaire mondial, 2014). Le profit annuel des vendeurs est aussi significativement et positivement influencé par la quantité de main-œuvre employé. Ceci traduit le fait que des valeurs élevées de ce paramètre, contribue à l'amélioration du profit net des vendeurs. L'effet significatif et positif de la quantité de main d'œuvre sur le profit net annuel du vendeur s'explique par le fait que les vendeurs n'achètent pas les fruits mais les collectent eux-mêmes ou bien recourent à la main d'œuvre familiale (principalement les enfants) non rémunérée. Le coût total des charges couvertes, bien que significatives, ont des effets négatifs sur le profit net annuel.

De manière générale, le profit des vendeurs est faible compte tenu de la non valorisation des fruits de $H$. barteri qui sont souvent abandonnés dans les formations végétales. En effet, les études effectuées par N'Diaye M et al., (2003) sur la valeur ajoutée provenant d'une meilleure valorisation des principaux fruits de cueillette consommés et commercialisés en Guinée tels que Vitellaria paradoxa, Adansonia digitata, Parkia biglobosa, etc, ont montré qu'il y a une différence significative entre les revenus nets tirés des produits transformés et ceux obtenus des produits non transformés. La valorisation des fruits de $H$. barteri et de leurs amendes peut constituer alors un moyen très important pour augmenter le niveau de revenu des vendeurs.

\section{Stratégies futures de conservation et de domestication \\ Malgré l'importance socio-} économique non négligeable de $H$. barteri dans la vie des populations locales du NordOuest du Bénin, les arbres de l'espèce ne bénéficient d'aucun soin particulier et ne sont gérées par aucun présumé propriétaire comme c'est le cas de certaines espèces telles que le karité et le néré dans la zone d'étude. Aussi, aucun plan d'aménagement préalablement établi n'existe.

Par ailleurs, des difficultés de régénération naturelle notées chez $H$. barteri (Agbogan et al., 2012 ; Sourou et al., 2016) et la récurrence dans les milieux savanicoles des facteurs de dégradation (Ouédraogo et al., 2006 ; Savadogo et al., 2007) limitent davantage le potentiel de régénération de l'espèce et la rend particulièrement vulnérable.

La gestion des populations de $H$. barteri à long terme est donc nécessaire pour améliorer les moyens de subsistance de la population locale. A cet effet, une sensibilisation efficace des villageois pour la protection de l'espèce dans les jachères et sa conservation in situ sur les flancs et les sommets des collines de l'Atakora pourraient permettre sa préservation future. Il est important de protéger les semis et les brins francs de pieds contre les feux et les coupes de bois, mais aussi les semenciers dispersés dans les champs.

Des études de reproduction sexuée (mode de reproduction, dormance, etc.) en 
milieu naturel et au laboratoire, de sélection variétale et des essais de multiplication végétative à faible coût notamment par marcottage (Noubissié et al., 2011), par induction de drageonnage (Meunier, 2008) ou par boutures de segments racinaires (Bellefontaine, 2013 ; Agbogan et al., 2014) de clones très productifs doivent être menées pour envisager une domestication progressive des clones performants dans les jardins de cases et un enrichissement éventuel des savanes boisées à $H$. barteri.

\section{Conclusion}

H. barteri est une ressource végétale indigène bien connue et utilisée par les populations du nord ouest de l'Atacora au Bénin. Les principaux organes de l'espèce à savoir les feuilles, les racines et l'écorce sont employés dans le traitement de plusieurs maladies/affections alors que les fruits sont essentiellement utilisés comme aliment par les populations locales de la zone d'étude. Ces différentes utilisations de $H$. barteri varient d'un groupe ethnique à un autre.

$H$. barteri n'a pas encore une valeur économique très remarquable à l'instar du baobab (Adansonia digitata), du néré (Parkia biglobosa) et du karité (Vitellaria paradoxa) par exemple, mais le revenu issu de la vente des fruits de $H$. barteri par les populations locales est loin d'être négligé. Le revenu annuel réalisé pour les fruits $H$. barteri est significativement influencé par la quantité totale de mains d'œuvre employée, la quantité totale de capital utilisée et l'ethnie du vendeur.

Une sensibilisation des paysans pour la protection de $H$. barteri dans les champs et jachères et sa conservation in situ sur les flancs et les sommets des collines de l'Atakora, combinée à une valorisation des fruits de l'espèce à travers leur transformation en boissons, jus, sirop ou en confiture à une grande échelle permettront non seulement aux paysans d'améliorer considérablement leur niveau de revenu mais aussi d'assurer la pérennité et la domestication de l'espèce.

\section{CONFLIT D'INTERET}

Les auteurs de ce manuscrit déclarent que l'article n'est sujet d'aucun problème ou d'intérêt concurrentiel.

\section{CONTRIBUTION DES AUTEURS}

Les auteurs de l'article ont participé chacun en ce qui le concerne à la mise au point du manuscrit. En effet, les objectifs du projet d'article, l'exécution du protocole, la rédaction et l'analyse statistique des données ont été effectués par BNS, l'investigateur principal, assisté de JY et CAINO. Le protocole donnant le sujet a été proposé par JY et NS. Enfin la lecture du manuscrit a été faite par CAINO et NS.

\section{REFERENCES}

Akoègninou A, Adjakidje V, Essou JP, Sinsin B, Yedomonhan H, Van Der Brug WJ, Van Der Maesen LJG. 2006. Flore Analytique du Bénin. Backhuys Publishers: Cotonou \& Wageningen; 1034.

Agbogan A, Tozo K, Wala K, Batawila K, Dourma M, Akpagana K. 2012. Abondance et structure des populations d'un fruitier spontané : Haematostaphis barteri Hook. F. dans deux sites rocheux en région soudanienne au Togo. Int. J. Biol. Chem. Sci., 6(6): 604-6048. DOI: http://dx.doi.org/10.4314/ijbcs.v6i6.31.

Agbogan A, Bammite D, Tozo K, Akpagana K. 2014. Contribution à la multiplication par graines et par bouturage de segments de tiges et de racines de trois fruitiers spontanés de la région des savanes au Togo : Haematostaphis barteri Hook. F., Lannea microcarpa Engl. \& K. Krause et Sclerocarya birrea (A. Rich.) Hochst. European Scientific Journal, 10(6): 195211. 
Agbogan A, Tozo K, Wala K, Bellefontaine R, Dourma M, Akpavi S, Woegan YA, Dimobe K, Akpagana K. 2015. Structure des populations de Sclerocarya birrea, Lannea microcarpa et Haematostaphis barteri au nord du Togo. Journal of Animal \& Plant Sciences, 25(2): 38713886. DOI: http://www.m.elewa.org/ JAPS.

Amoo IA, Lajide L. 1999. Chemical composition and nutritive significance of underutilised Haematostaphis barteri fruit. Rivista Italiana delle Sostanze Grasse, 76(10): 441-442.

Amouzou KS, Adaké B, Batawila K, Wala K, Akpavi S, Kanda M, Odah K, Titrikou KK, Butaré I, Bouchet P, Akpagana K. 2006. Études biochimiques et évaluation des valeurs nutritionnelles de quelques espèces alimentaires mineures du Togo. Acta Botanica Gallica, 153(2): 147-152. DOI:10.1080/12538078.2006.10515533.

Arbonnier M. 2002. Arbres Arbustes et Lianes des Zones Sèches d'Afrique de l'Ouest, (2è edn). CIRAD-MNHN ; 573.

Asase A, Oteng-yeboah A, Odamtten T, Simmonds M, 2005. Ethnobotanical study of some Ghanaian anti-malarial plants. Journal of Ethnopharmacology, 99(2): 273-279. DOI : http://dx.doi.org/10.1016/j.jep.2005.02.0 20 .

Assogbadjo AE. 2006. Importance socioéconomique et étude de la variabilité écologique, morphologique, génétique et biochimique du baobab (Adansonia digitata L.) au Bénin. Thèse de doctorat. Faculty of Bioscience Engineering, Ghent University, Belgium, p.213.

Assogbadjo AE, Glèlè Kakaï R, Adjallala FH, Azihou AF, Vodouhê GF, Kyndt T, Codjia JTC. 2010. Ethnic differences in use value and use patterns of the threatened multipurpose scrambling shrub (Caesalpinia bonduc L.) in Benin. Journal of Medicinal Plants Research,
5(9): $\quad$ 1549-1557. DOI: http://www.academicjournals.org/JMPR. Balogoun I, Saïdou A, Ahoton EL, AmadjI LG, Ahohuendo CB, Adebo IB, Babatounde S, Chougourou D, Adoukonou-Sagbadja H, Ahanchédé A. 2014. Caractérisation des systèmes de production à base d'anacardier dans les principales zones de culture au Bénin. Agronomie Africaine, 26(1): 9-22.

Bellefontaine R, Ferradous A, Alifriqui M, Bouzoubaa Z, Ky-Dembele C, Nsibi R, Le Bouler H, Meunier Q. 2013. Multiplication végétative d'arganiers par greffes, drageons et boutures de segments racinaires. In Actes du ler Congrès international de l'arganier, Agadir, 15-17 décembre 2011, 379-388. Agadir: INRA-ANDZOA. DOI: http://www.inra.org.ma/Docs/actesargan ier/arganier379388.pdf.

Boampong JN, Karikari AA, Ameyaw EO. 2015. In vivo antiplasmodial and in vitro antioxidant properties of stem bark extracts of Haematostaphis barteri. Asian Pac. J. Trop. Biomed., 5(6): 446-450. DOI: http://dx.doi.org/10.1016/j.apjtb. 2015.02.002

Bowe C, Haq N. 2010. Quantifying the global environmental niche of an underutilized tropical fruit tree (Tamarindus indica) using herbarium records. Agriculture, Ecosystems and Environment, 139(1-2): 51-58. DOI: http://dx.doi.org/10.1016/ j.agee.2010.06.016.

Dagnelie P. 1998. Statistiques Théoriques et Appliquées. de Boeck et Larcier: Bruxelles, Belg.

Dan Guimbo I, Barage M, Douma S. 2012. Etudes préliminaires sur l'utilisation alimentaire des plantes spontanées dans les zones périphériques du parc $\mathrm{W}$ du Niger. Int. J. Biol. Chem. Sci., 6(6): 4007-4017. DOI : http://dx.doi.org/ 10.4314/ijbcs.v6i6.12. 
Eromosele CO, Eromosele IC. 2002. Fatty acid compositions of seed oils of Haematostaphis barteri and Ximenia americana. Bioresource Technology, 82(3): 303-304. DOI: http://dx.doi.org/10.1016/s09608524(01)00179-1.

Eyana KA. 2007. Les anacardiaceae du Togo : Études Botaniques, Écologiques et Propriétés antifongiques. Thèse de doctorat, Université de Reims, p.183.

Eyog Matig, Gaoué OG, Dossou B. 2002. Réseau «Espèces Ligneuses Alimentaires». Compte rendu de la première réunion du Réseau tenue 11-13 décembre 2000 au CNSF Ouagadougou, Burkina Faso 241: Institut International des Ressources Phytogénétiques.

Gnanglè PC, Afouda Yabi J, Rosaine Yegbemey N, Romain Glèlè Kakaï, Sokpon N. 2012. Rentabilité économique des systèmes de production des parcs à karité dans le contexte de l'adaptation au changement climatique du nord-bénin. African Crop Science Journal, 20(2): 589-602. DOI : http://dx.doi.org/10.4314/ijbcs.v6i1.13.

Gouwakinnou GN, Mette Lykke A, Assogbadjo AE, Sinsin B. 2011. Local knowledge, pattern and diversity of use of Sclerocarya birrea. Journal of Ethnobiology and Ethnomedicine, 7(1). DOI: http://dx.doi.org/10.1186/17464269-7-8.

INSAE-RGPH. 2002. Recensement Général de la Population et de l'Habitat: Synthèses des Analyses. INSAE-RGPH : Cotonou, Bénin ; 47.

Kubmarawa D, Adnenyang IFH, Magomy AM. 2009. Proximate composition and amino acid profile of two nonconventional leafy vegetables (Hibiscus cannabinus and Haematostaphis barteri). African Journal of Food Science, 3(9): 233-236. DOI: http://www.academicjournals.org/AJB.
Makalao MM, Savadogo A, Zongo C, et Traore AS. 2015. Composition nutritionnelle de 10 fruits sauvages consommés dans trois départements du Tchad. Int. J. Biol. Chem. Sci., 9(5): 2385-2400. DOI http://dx.doi.org/10.4314/ijbcs.v9i5.11

MEHU 2002. Stratégie Nationale et Plan d'Action pour la Conservation de la Diversité Biologique au Bénin. MEHU : Cotonou, Bénin ; 144.

Meunier Q, Bellefontaine R, Monteuuis O. 2008. La multiplication végétative d'arbres et arbustes médicinaux au bénéfice des communautés rurales d'Ouganda. Bois et Forêts des Tropiques, 295(2): 71-82. DOI : http://bft.cirad.fr/cd/BFT_296_7182.pdf?.

N'Diaye M, Kéita FB, Martin P. 2003. Principaux fruits de cueillette consommés et commercialisés en Guinée. Fruits, 58(2): 89-98. DOI: http://dx.doi.org/10.1051/fruits:2002040.

Noubissié Tchiagam JB, Ndzie JP, Bellefontaine R, Mapongmetsem PM. 2011. Multiplication végétative de Balanites aegyptiaca (L.) Del., Diospyros mespiliformis Hochst. ex. A.Rich. et Sclerocarya birrea (A. Rich.) Hochst. Au nord du Cameroun. Fruits, 66(5) : 327-341. DOI: http://dx.doi.org/10.1051/fruits/2011047.

Ouédraogo A, Thiombiano A, Hahn-Hadjali K, Guinko S. 2006. Diagnostic de l'état de dégradation des peuplements de quatre espèces ligneuses en zone soudanienne du Burkina Faso. Sécheresse, 17(4): 485-491. DOI: 10.1684/sec.2006.0058.

Programme Mondial Alimentaire, Service de l'Analyse de la Sécurité Alimentaire. 2014. Analyse Globale de la Vulnérabilité et de la Sécurité Alimentaire (AGVSA), République du 
Bénin. Consulté le 02 mai 2014 sur le site http://www.wfp.org/food-security.

Savadogo P, Tigabu M, Sawadogo L, Oden PC. 2007. Woody species composition, structure and diversity of vegetation patches of a Sudanian savanna in Burkina Faso. Bois et Forêts des Tropiques, 294(4): 520. DOI : http://bft.cirad.fr/cd/ BFT_294_5-20.pdf.

Sourou BN, Ouinsavi CAIN and Sokpon N. 2016. Ecological Structure and Fruit Production of Blood Plum (Haematostaphis barteri Hook. F) Subpopulations in Benin. International Journal of Plant \& Soil Science, 9(2): 112. DOI: 10.9734/IJPSS/2016/22059.

Tadzabia K, Maina HM, Maitera ON, Ezekiel JS. 2013. Evaluation of phytochemical and elemental contents of Haematostaphis barteri leaves and stem bark in Hong local government area of Adamawa state, Nigeria. Journal of Chemical and Pharmaceutical Research, 5(9):150-156. DOI: www.jocpr.com.

TchabI VI, Adechi KO. 2014. Occupation des terres, typologie et structure des faciès de végétation à Vitellaria paradoxa (Gaertn F.) de la Commune de Toukountouna au Nord-Bénin. Int. J. Biol. Chem. Sci., 8(4): 1684-1696. DOI : http://dx.doi.org/10.4314/ijbcs.v8i4.29

Umaru HA, Adamu R, Dahiru D, Nadro MS. 2007. Levels of antinutritional factors in some wild edible fruits of Northern Nigeria. African Journal of Biotechnology, 6(16):1935-1938. DOI: http://www.academicjournals.org/AJB.

World Agroforestry Centre. 2008. Transforming Lives and Landscapes. Strategy. 2008-2015, Nairobi, Kenya. 